\title{
Mental Health and Benzodiazepine Use Among Patients on Chronic Opioid Therapy
}

\author{
Abigail Y. Smith, MD, Julienne K. Kirk, PharmD, Paul F. Smith, BS, and \\ Brian J. Wells, MD, PhD
}

Introduction: Higher daily doses of opioids as well as co-prescription of benzodiazepines have been associated with risk of overdose. The current study characterizes prescribing patterns in a family medicine practice with regard to patient mental health diagnoses, benzodiazepine prescriptions, morphine milligram equivalent opioid dose, and patient demographics.

Methods: Patients on chronic opioid therapy were studied in 2018 and 2019. Mental health diagnoses, opioid dose, benzodiazepine prescriptions and demographic characteristics were extracted from the electronic health record. Data were compared between years and logistic regression was used to determine which patient characteristics were associated with likelihood of decreased opioid dose.

Results: A total of 387 patients were prescribed chronic opioid therapy in 2018, and 231 in 2019. In 2018, $49.9 \%$ of patients prescribed chronic opioids had mental health diagnoses. In 2019, this proportion rose to $92.2 \%$. In 2019,205 of the original 387 patients were still with the practice but were not prescribed chronic opioids. Among the factors studied, psychiatric diagnosis and higher opioid dose were associated with a significantly lower likelihood of tapering doses.

Discussion: As practices taper or de-prescribe opioids, or implement harm reduction methods such as de-prescribing benzodiazepines, it is important to understand patient characteristics and their relationship to success with tapering. This study adds to the evidence that odds of successfully tapering opioids may be significantly impacted by patients' mental health diagnosis and opioid dose. ( $\mathrm{J}$ Am Board Fam Med 2021;34:99-104.)

Keywords: Benzodiazepines, Chronic Disease, Electronic Health Records, Family Medicine, Harm Reduction, Morphine, Logistic Models, Mental Health, Opioids, Pain Management

\section{Introduction}

Opioid use is common among patients with mental health disorders in the United States. One study reported that $51.4 \%$ of total opioid prescriptions are dispensed to those with mental health disorders. Among adults with a mental health disorder, $18.7 \%$ were prescribed an opioid, versus $5 \%$ of patients without a mental health disorder. ${ }^{1}$ Use of opioids

This article was externally peer reviewed.

From the Department of Family and Community Medicine, Wake Forest School of Medicine, Winston-Salem, NC (AYS, JKK, BJW); Department of Information Technology Services, Wake Forest School of Medicine, Winston-Salem, NC (PFS); Division of Public Health Sciences, Department of Biostatistical Sciences, Center for Biomedical Informatics, Winston-Salem, $\mathrm{NC}(\mathrm{BJW})$.

Funding: None.

Conflict of interest: None.

Corresponding author: Julienne K. Kirk, PharmD, CDE, BCPS, Department of Family and Community Medicine, Medical Center Boulevard, Wake Forest School of Medicine, Wake Forest Baptist Health, Winston-Salem, NC 27157 (E-mail: jkirk@wakehealth.edu). for greater than 30 days has been linked with increased risk of depression. ${ }^{2}$ Conversely, patients with a depression diagnosis have a higher odds of being prescribed an opioid. ${ }^{3,4}$ Depressive symptoms in patients with chronic pain are associated with higher dropout and relapse rates from tapering of opioid medication. ${ }^{5}$

Increasing dose of opioids, measured in morphine milligram equivalents (MME) per day, is associated with greater risk of overdose, with patients on doses of $100 \mathrm{MME}$ or greater per day at a 9-fold increased risk compared with patients on the lowest doses. ${ }^{6}$ Co-prescription of opioids and sedative-hypnotics has been found to be associated with increased risk of overdose. ${ }^{6}$ A benzodiazepine prescription has also been shown to be a risk factor for opioid prescription. ${ }^{7}$

The increase in opioid-related harms in the United States has led the Centers for Disease Control and Prevention to publish the Guideline for 
Prescribing Opioids for Chronic Pain, which brings widespread awareness to the issue. ${ }^{8}$ This has led to a decrease in the overall number of opioid prescriptions. In addition, it has decreased situations that pose risk for harm, such as increased daily MME doses of greater than 90 and overlapping benzodiazepine and opioid prescriptions. $^{9}$

In 2018 and 2019, all providers in the Department of Family Medicine were offered medicationassisted therapy (MAT) waiver training. In November 2019, we assembled a MAT clinic within our outpatient practice. The MAT clinic included any patient referral for consideration for opioid tapering and Suboxone therapy. The residency program had multiple didactic sessions regarding safe opioid prescribing, opioid and benzodiazepine tapering, and harm reduction. Providers received a list of prescriptions written during the 2018 and 2019 calendar years that included opioid and benzodiazepine detailed prescribing information (ie, dosage, frequency, MME). We distributed reports in January 2019 and 2020 encompassing the entire previous year. Our clinical pharmacists contacted all high-risk patients who did not yet have a naloxone prescription and ensured that they received a prescription and a significant other was instructed on naloxone use.

The purpose of the present study is to explore the characteristics of patients receiving chronic opioid prescriptions in a family medicine residency clinic, and to evaluate whether and how these characteristics changed from 2018 to 2019. The concomitant presence of psychiatric diagnoses, concomitant prescriptions for benzodiazepines, and the distribution of opioid doses among family medicine patients were evaluated and compared year over year.

\section{Methods}

\section{Patient Selection and Practice Characteristics}

This study was conducted in an academic Department of Family and Community Medicine associated with a residency program. The department employs 30 residents, 52 physician faculty, and 5 advanced-practice providers. The practice saw 14,166 individual patients in 2018 and 14,828 individual patients in 2019. A MAT clinic operates alongside the primary care practice at the residency clinic. Patients $\geq 18$ years of age who received at least 1 outpatient prescription for an oral opioid medication from a family medicine provider in 3 separate months in the relevant calendar year were considered to be on chronic opioid therapy and were included in the study. The study included a cross-sectional characterization of the patients who met the chronic opioid definition in 2018, and again from 2019. Patient characteristics were defined according to a reference date of December 31 of the associated year. Opioid medications were classified based on therapeutic class definitions and reviewed by a clinical pharmacist. Tramadol was excluded due to inability to calculate MME. Benzodiazepine treatment was simply defined as at least 1 prescription during the relevant calendar year. Mental health categories were based on a modification of ICD-10 groupings created by the World Health Organization. ${ }^{10}$ The list was edited to exclude organic and sexual disorders, those secondary to the use of psychoactive substances, those related to substance abuse, and learning and developmental disabilities.

\section{Data Collection}

Data for the project was extracted from the Clarity (Epicare, Verona, WI) relational database that serves as a repository for electronic health record (EHR) data and is updated on a nightly basis. Among patients on chronic opioid therapy, mental health diagnoses were extracted from the encounter diagnoses in the associated year.

The daily MME per patient was calculated as a mean across the entire year in the following manner: sum of the total MME contained in all prescriptions divided by 365 . The investigators removed discontinued prescriptions with a discontinuation reason of "written in error," and prescriptions with start dates outside the study time frame. Anecdotally, providers sometimes write 1 or 2 extra postdated prescriptions for patients who are seen less frequently than once a month. The investigators excluded extra prescriptions written at the end of the calendar year that would be filled in the subsequent year.

\section{Data Analysis}

Summary statistics were calculated for patients included in the 2018 and 2019 cohort, respectively. Differences in the patient characteristics were compared in a univariate fashion using the chi-squared test, $t$-test, and rank sum as appropriate. In addition, the authors examined the change in opioid dosage, mental health diagnoses, and concomitant benzodiazepine prescriptions among patients included in the 2018 cohort. Patients from the 2018 cohort without 
at least 1 in-person visit in the Department of Family and Community Medicine in 2019 were assumed to have left the practice and were considered lost to follow-up.

Given the department's active attempts to reduce chronic opioid prescribing, the investigators were interested in determining the patient characteristics associated with successful de-escalation of opioid therapy. Adjusted analyses were conducted using logistic regression with unsuccessful de-escalation as the independent variable, including the following dependent variables: gender (male vs female), race (white vs nonwhite), and category of MME per day in $2018(<50,50$ to $89, \geq 90)$. Statistical analyses were performed in $\mathrm{R}$ version 3.4.0 (Vienna, Austria). The variance inflation factor (VIF) was used to assess potential multicollinearity between independent variables. An interaction term was applied between psychiatric diagnosis and benzodiazepine prescription.

The present study was approved by the Wake Forest School of Medicine Institutional Review Board.

\section{Results}

Three hundred and eighty-seven patients in the family medicine practice met the definition for chronic opioid use in 2018, which decreased to 231 in 2019 (Table 1). Of the patients on chronic opioid therapy in $2018,49.9 \%$ had 1 or more mental health diagnoses. In 2018, depression and anxiety were most common at $26.1 \%$ and $28.2 \%$, respectively. In $2019,92.2 \%$ of patients on chronic opioid therapy had a mental health diagnosis, with depression and anxiety at $66.7 \%$ and $60.6 \%$, respectively.

$31.3 \%$ of patients on chronic opioid therapy in 2018 also had 1 or more prescriptions for a benzodiazepine. This proportion remained relatively unchanged in 2019 at $33.3 \%$. In 2018, 10.3\% of patients with chronic opioid prescriptions were prescribed 50 to $89.9 \mathrm{MME}$ per day, and $8.0 \%$ of patients were prescribed $\geq 90 \mathrm{MME}$ per day. In $2019,14.3 \%$ had an average daily dose of 50 to $89.9 \mathrm{MME}$, and $13.0 \%$ had an average daily dose $\geq 90$ MME.

To further explore changes from year to year, we tracked patient movement between categories (Table 2 and Table 3). Among the 387 patients on chronic opioids in 2018, 205 were no longer on opioids in 2019. This includes 18 of 71 (25.4\%) of those who were on greater than $50 \mathrm{MME}$ per day
Table 1. Demographic Characteristics, Mental Health Diagnoses, Opioid Daily Dose, and Benzodiazepine Prescriptions Among Patients on Chronic Opioid Therapy in 2018 and 2019

\begin{tabular}{|c|c|c|c|}
\hline & 2018 & 2019 & $P$ \\
\hline $\mathrm{N}$ & 387 & 231 & \\
\hline Age, mean (SD) & $60.60(12.59)$ & $59.75(11.57)$ & .404 \\
\hline Male gender, n (\%) & $140(36.2)$ & $72(31.2)$ & .238 \\
\hline Race, n (\%) & & & .053 \\
\hline $\begin{array}{l}\text { Black or African } \\
\text { American }\end{array}$ & $154(39.8)$ & $73(31.6)$ & \\
\hline White or Caucasian & $229(59.2)$ & $152(65.8)$ & \\
\hline Other & $4(1.0)$ & $6(2.6)$ & \\
\hline Ethnicity, n (\%) & & & .245 \\
\hline Hispanic or Latino & $2(0.5)$ & $4(1.7)$ & \\
\hline Not Hispanic or Latino & $384(99.2)$ & $227(98.3)$ & \\
\hline Patient refused & $1(0.3)$ & $0(0.0)$ & \\
\hline $\begin{array}{l}\text { Any psychiatric } \\
\text { diagnosis, } n(\%)\end{array}$ & $193(49.9)$ & $213(92.2)$ & $<.001$ \\
\hline Depression, n (\%) & $101(26.1)$ & $154(66.7)$ & $<.001$ \\
\hline Anxiety, n (\%) & $109(28.2)$ & $140(60.6)$ & $<.001$ \\
\hline Bipolar, n (\%) & $21(5.4)$ & $20(8.7)$ & .163 \\
\hline Adjustment, n (\%) & $43(11.1)$ & $70(30.3)$ & $<.001$ \\
\hline PTSD, n (\%) & $6(1.6)$ & $12(5.2)$ & .018 \\
\hline Other, n (\%) & $24(6.2)$ & $39(16.9)$ & $<.001$ \\
\hline MME daily dose & & & .03 \\
\hline$\geq 90$ MME, n (\%) & $31(8.0)$ & $30(13.0)$ & \\
\hline 50 to $89.9 \mathrm{MME}, \mathrm{n}(\%)$ & $40(10.3)$ & $33(14.3)$ & \\
\hline <50MME, n (\%) & $316(81.7)$ & $168(72.7)$ & \\
\hline Benzodiazepine Rx n (\%) & $121(31.3)$ & $77(33.3)$ & .657 \\
\hline
\end{tabular}

MME, morphine milligram equivalent; SD, standard deviation; PTSD, post-traumatic stress disorder.

in 2018, and 187 of $316(59.2 \%)$ of those on less than 50 MME per day. Of the 121 patients co-prescribed a benzodiazepine in 2018, 54 patients were not prescribed a benzodiazepine in 2019, and 46 were no longer on chronic opioids.

Of the 193 patients in the 2018 data set with a mental health diagnosis, 32 did not have a mental health diagnosis documented the following year, and 73 were no longer on chronic opioids. Of the 184 patients without a psychiatric diagnosis in 2018, 38 had a diagnosis in 2019.

The VIF values for each of the dependent variables failed to show evidence of significant multicollinearity (all VIF values < 1.5). The interaction term between psychiatric diagnosis and benzodiazepine prescription was not statistically significant and was removed from the model. The adjusted results (Table 4) show that the presence of a 
Table 2. Transition of Patients between Benzodiazepine Prescription Categories from 2018 to 2019, Classified by MME Daily Dose Category

\begin{tabular}{|c|c|c|c|c|c|c|}
\hline \multirow[b]{2}{*}{2018 Category } & \multicolumn{6}{|c|}{2019 Category } \\
\hline & $\begin{array}{c}<50 \mathrm{MME} \\
\text { without } \\
\text { Benzodiazepine }\end{array}$ & $\begin{array}{c}\geq 50 \mathrm{MME} \\
\text { without } \\
\text { Benzodiazepine }\end{array}$ & $\begin{array}{l}<50 \mathrm{MME} \text { with } \\
\text { Benzodiazepine }\end{array}$ & $\begin{array}{l}\geq 50 \mathrm{MME} \text { with } \\
\text { Benzodiazepine }\end{array}$ & $\begin{array}{l}\text { No } \\
\text { Visits }\end{array}$ & $\begin{array}{l}\text { Not on Chronic } \\
\text { Opioids }\end{array}$ \\
\hline $\begin{array}{l}<50 \text { MME without } \\
\quad \text { benzodiazepine }(n=226)\end{array}$ & 40 & 13 & 19 & 7 & 2 & 145 \\
\hline $\begin{array}{l}\geq 50 M M E \text { without } \\
\text { Benzodiazepine }(n=40)\end{array}$ & 14 & 5 & 3 & 4 & 0 & 14 \\
\hline $\begin{array}{l}<50 \text { MME with } \\
\quad \text { Benzodiazepine }(\mathrm{n}=90)\end{array}$ & 31 & 5 & 8 & 4 & 0 & 42 \\
\hline $\begin{array}{l}\geq 50 M M E \text { with } \\
\quad \text { Benzodiazepine }(\mathrm{n}=31)\end{array}$ & 13 & 5 & 5 & 3 & 1 & 4 \\
\hline Total $(\mathrm{n}=387)$ & 98 & 28 & 35 & 18 & 3 & 205 \\
\hline
\end{tabular}

MME, morphine milligram equivalent.

psychiatric diagnosis during an encounter in 2018 was associated with a higher odds of remaining on chronic opioid therapy at the same or higher dosage (odds ratio [OR], 2.73; 95\% CI, 1.71-4.37) in 2019. Similarly, patients on $\geq 90$ MME per day (OR 2.75 ; 95\% CI, 1.15-6.59) or 50-89 MME (OR, 2.18 ; $95 \%$ CI , 1.05-4.50) were more likely to remain on the same or higher MME dosage in 2019.

\section{Discussion}

There is a well-documented association between diagnosis with a mental health disorder and prescription of opioid medications. ${ }^{1-3,11}$ In the present study population of patients on chronic opioid therapy, $49.9 \%$ had 1 or more mental health diagnoses in the initial study year. A previous study found that over half of opioid prescriptions go to patients with mental health diagnoses. ${ }^{1}$ In the second year examined, the proportion of patients on chronic opioids who had a mental health diagnosis increased significantly, to $92.2 \%(P<.001)$. Patients who were not successfully tapered to a lower dose category had 2.73 times higher odds of having a psychiatric diagnosis. The increase in absolute number of patients in this category is consistent with the finding of 1.8 times the odds of prescription of opioid medications during a visit when a patient has a depression diagnosis in addition to pain. ${ }^{4}$ The present study findings show a high percentage of patients in a primary care practice with mental health diagnoses were not tapered. This adds to the evidence that patients with depressive symptoms have a higher risk of failure or relapse in opioid-tapering programs. ${ }^{5}$

Table 3. Transition of Patients between Mental Health Diagnosis Categories from 2018 to 2019, Classified by MME Daily Dose Category

\begin{tabular}{|c|c|c|c|c|c|c|}
\hline \multirow[b]{2}{*}{2018 Category } & \multicolumn{6}{|c|}{2019 Category } \\
\hline & $\begin{array}{l}<50 \text { MME with- } \\
\text { out Psych Dx }\end{array}$ & $\begin{array}{l}\geq 50 \text { MME with- } \\
\text { out Psych Dx }\end{array}$ & $\begin{array}{c}<50 M M E \text { with } \\
\text { Psych Dx }\end{array}$ & $\begin{array}{l}\geq 50 \mathrm{MME} \text { with } \\
\text { Psych Dx }\end{array}$ & $\begin{array}{l}\text { No } \\
\text { Visits }\end{array}$ & $\begin{array}{l}\text { Not on Chronic } \\
\text { Opioids }\end{array}$ \\
\hline $\begin{array}{l}<50 \text { MME without psych } \\
\text { diagnosis }(\mathrm{n}=165)\end{array}$ & 14 & 3 & 26 & 3 & 2 & 117 \\
\hline $\begin{array}{l}\geq 50 \text { MME without psych } \\
\text { diagnosis }(\mathrm{n}=29)\end{array}$ & 4 & 1 & 5 & 4 & 0 & 15 \\
\hline $\begin{array}{l}<50 \text { MME with psych } \mathrm{dx} \\
\quad(\mathrm{n}=151)\end{array}$ & 15 & 6 & 43 & 17 & 0 & 70 \\
\hline $\begin{array}{l}\geq 50 M M E \text { with psych } \\
\text { diagnosis }(\mathrm{n}=42)\end{array}$ & 4 & 7 & 22 & 5 & 1 & 3 \\
\hline Total $(\mathrm{n}=387)$ & 37 & 17 & 96 & 29 & 3 & 205 \\
\hline
\end{tabular}

MME, morphine milligram equivalent. 
Table 4. Odds of Remaining in the Same or Higher MME per Day Dose Category Based on Patient Characteristics

\begin{tabular}{lcc}
\hline & $\begin{array}{l}\text { Odds } \\
\text { Ratio }\end{array}$ & 95\% CI \\
\hline White versus nonwhite & 1.62 & 1.00 to 2.61 \\
Psychiatric diagnosis versus none & 2.73 & 1.71 to 4.37 \\
$\begin{array}{l}\text { Benzodiazepine prescription versus } \\
\quad \text { none }\end{array}$ & 1.17 & 0.70 to 1.95 \\
$\begin{array}{l}\text { Gender male versus female } \\
\text { 50 to 89.9 MME per day versus }\end{array}$ & 0.67 & 0.42 to 1.09 \\
$\quad$ < 50 MME per day & 2.18 & 1.05 to 4.50 \\
$\geq 90 \mathrm{MME}$ per day versus $<50 \mathrm{MME}$ & 2.75 & 1.52 to 6.59 \\
$\quad$ per day & & \\
\hline
\end{tabular}

MME, morphine milligram equivalent; CI, confidence interval.

Clinicians are using increasing caution with the co-prescription of opioids and benzodiazepines, given data on overdose. ${ }^{6}$ In this study, around one third of patients on chronic opioid therapy also had a benzodiazepine prescribed, which is lower than the $46 \%$ and $74 \%$ found in other recent studies. ${ }^{6,12}$ This proportion remained essentially the same from 2018 to 2019 (31.3\% to $33.3 \%$; $P=.657)$, and the odds of a benzodiazepine prescription were not significantly different between patients who were and were not tapered off of opioids, similar to findings in another recent study. ${ }^{13}$ Unlike the study by Buonora et $a l,{ }^{13}$ we did not find a significant difference in likelihood of tapering based on gender or race. In the present study, higher opioid dose was associated with a greater likelihood of remaining in the same dose category between 2018 and 2019, meaning that patients on higher doses were less likely to be tapered down or off of opioids, and patients on lower doses accounted for the majority patients who were no longer on chronic opioids in the second year studied. A study by Tong et al. ${ }^{7}$ reported qualitative data from interviews with clinicians, in which barriers to tapering were identified. Among those cited were lack of time, lack of control over patients' outside sources of opioids such as other prescribers, and difficulty justifying tapering in patients on stable, long-term doses. ${ }^{7}$ This analysis allowed comparison to existing data on opioidprescribing practices. The present study also provided year over year comparisons to quantify the results of efforts in education on opioid tapering and harm reduction in the department. The results of the present study highlight the need for particular attention to patients with mental health diagnoses when attempting to taper opioid doses. Future studies are needed to further delineate effective support and care of the patient with a mental health diagnosis who is being weaned from opioids.

\section{Study Limitations}

There are limitations in the analyses that could have biased the results. The calculation of MME was averaged across the entire year, but it is possible that patients were not taking opioid medication continuously throughout the entire year. In addition, opioid prescriptions written outside of the study practice were not captured with these data. Our analysis was based on prescriptions, not fill data or patient reports of taking medications, which may also bias results. We did not collect type of pain treated or severity. This analysis is based on medical record review, with its inherent flaws and potential data inaccuracies. We did not track patients who left the practice, so a detailed analysis of their outcomes is not possible with this data set. However, this number is limited as evidenced by 3 patients from 2018 who were no longer in the study in 2019. Although education on opioid prescribing was provided, this was not standardized, making it impossible to ensure that all providers in the study received equivalent education. As the present study utilized a cross-sectional design, we are only able to speculate regarding cause-effect relationships in the trends observed. We attempted to mitigate this by comparing individual patients year to year. This study was performed in a single family medicine practice, which may limit generalizability.

With these limitations in mind, this study does provide information on deprescribing and tapering patterns that builds on the current available literature. The complicated interplay between opioid therapy and mental health and the implications for tapering or weaning warrants further study.

To see this article online, please go to: http://jabfm.org/content/ 34/1/99.full.

\section{References}

1. Davis MA, Lin LA, Liu H, Sites BD. Prescription opioid use among adults with mental health disorders in the United States. J Am Board Fam Med 2017;30:407-17.

2. Scherrer JF, Salas J, Copeland LA, et al. Prescription opioid duration, dose, and increased risk of depression 
in 3 large patient populations. Ann Fam Med 2016; 14:54-62.

3. Olsen Y, Daumit GL, Ford DE. Opioid prescriptions by U.S. primary care physicians from 1992 to 2001. J Pain 2006;7:225-35.

4. Lin SX, Patel K, Younge RG. Opioid medications prescribing and the pain-depression dyad in primary care: analysis of 2014-2015 National Ambulatory Medical Care Survey (NAMCS) Data. J Am Board Fam Med 2019;32:614-8.

5. Heiwe S, Lonnquist I, Kallmen H. Potential risk factors associated with risk for drop-out and relapse during and following withdrawal of opioid prescription medication. Eur J Pain 2011;15:966-70.

6. Dunn KM, Saunders KW, Rutter CM, et al. Overdose and prescribed opioids: associations among chronic non-cancer pain patients. Ann Intern Med 2010;152:85-92.

7. Tong ST, Hochheimer CJ, Brooks EM, et al. Chronic opioid prescribing in primary care: factors and perspectives. Ann Fam Med 2019;17:200-6.

8. Dowell D, Haegerich TM, Chou R. CDC guideline for prescribing opioids for chronic pain-United
States, 2016. MMWR Recomm Rep 2016;65: 1-49.

9. Bohnert ASB, Guy GP, Losby JL. Opioid prescribing in the United States before and after the Center for Disease Control and Prevention's 2016 opioid guideline. Ann Intern Med 2018;169:367-75.

10. World Health Organization. The ICD-10 classification of mental and behavioural disorders: clinical descriptions and diagnostic guidelines. 2020. Available from: https://www.who.int/classifications/icd/ en/bluebook.pdf. Accessed June 26, 2020.

11. Sullivan MD, Edlund MJ, Steffick D, Unutzer J. Regular use of prescribed opioids: association with common psychiatric disorders. Pain 2005;119:95103.

12. Sun EC, Dixit A, Humphreys K, Damall BD, Baker LC, Mackey S. Association between concurrent use of prescription opioids and benzodiazepines and overdose: retrospective analysis. BMJ 2017;356:1-7.

13. Buonora M, Perez HR, Heo M, Cunningham CO, Starrels JL. Race and gender are associated with opioid dose reduction among patients on chronic opioid therapy. Pain Med 2019;20:1519-27. 